\title{
Cell Growth Models Using Reaction Schemes: Batch Cultivation
}

\author{
Svetoslav Markov \\ Institute of Mathematics and Informatics \\ Bulgarian Academy of Sciences \\ Sofia, Bulgaria \\ smarkov@bio.bas.bg
}

Received: 27 October 2013, accepted: 30 December 2013, published: 18 January 2014

\begin{abstract}
Simple structured mathematical models of bacterial cell growth are proposed. The models involve fractions of bacterial cells related to their physiological phases. Reaction schemes involving the biomass of the cell fractions, the substrate and the product are proposed in analogy to reaction schemes in enzyme kinetics. Applying the mass action law these reaction schemes lead to dynamical models represented by systems of ODE's. All parameters of the models are rate constants with clear biological or biochemical meaning. The proposed models generalize classical bacterial growth models and offer more flexible tools for modelling and control of biotechnological processes. In this paper the study is focused on batch cultivation models. We formulate a hypothesis that cell growth models can be entirely based on reaction schemes.
\end{abstract}

\section{INTRODUCTION}

Bacterial cell growth involves reaction steps such as cell phase transition, reproduction and mortality. We propose reaction schemes combining such reaction steps. We then apply the mass action law to formulate simple dynamical models of bacterial growth which account for the physiological states of bacterial cells. The cells are classified into fractions (compartments) related to their physiological states.

Classical bacterial cell growth models make use of the assumption that all cells are in the same physiological state at a given moment $t$. Therefore these models involve a single variable representing the biomass (population density) of the organisms. It has been recognized since long that most classical cell growth models describe adequately real processes under favorable environmental conditions, whenever organisms are steadily at $\log$ (exponential) phase when the cells actively divide and grow at a maximal rate. However, the environment in bio-reactors may be perturbed by various factors and the cells change their physiological state.

Under perturbed conditions classical cell growth models may fail to reflect adequately the dynamics of the bio-processes. Modifications of these models in various directions have been proposed in the literature. A wellstudied direction is to allow some of the parameters in the model, such as the "specific growth rate", to depend on the nutrient substrate and on other quantities such as temperature, $\mathrm{pH}$ etc. [3]. Another modelling directionnot so well explored-is based on the assumption that the bacterial cells are not simultaneously in the same physiological state (as assumed in classical models). Such models are named "structured" [9].

\section{A. Structured cell growth models}

An important feature of bacteria is their physiological state/phase (lag, log, stationary, dead). The physiological state depends on the environmental conditions for the cell population. We assume that not all cells are simultaneously in the same state. For example, the log (exponential) phase is the time period when the cells reproduce themselves. It is realistic to assume that not all cells are in log state at a given moment $t$. Assume that the environmental conditions have been unfavorable and then have rapidly improved so that the cells start 
changing their physiological state from lag to log phase. Some cells that are in better physiological condition than others and will start to reproduce earlier. There could exist cells that are not able to reproduce at all (genetically damaged, defect cells), but still viable (in lag phase). We assume that certain fractions of cells may react differently under similar conditions changing dynamically their physiological states (say, from lag to log phase and conversely). Thus organisms can be classified into two (or more) fractions corresponding to their physiological states and degree of adaptation ability. Mathematically this means to assign different phase variables for the biomass (population density) of the respective fractions of organisms and to study the dynamics of these fractions as separate cell populations.

Structured models inspired by models of phytoplankton [2] have been proposed and used in [4], [8], [9]. We accept the idea of structured models in so far that cell population is subdivided into fractions. In addition our modelling approach is tightly related to enzyme kinetics. This contributes to more clarity in the biological interpretation of the partaking terms in the models. Our approach aims to assign clear biological meaning to all parameters involved. In particular, no parameters depend on other quantities, that is, only numeric parameters are used. We propose reaction schemes for the transition dynamics of the cell fractions and the substrate/products involved. We believe that such reaction schemes will be helpful in understanding the biological mechanisms of the cell growth dynamics and, in particular, in understanding the biological nature of the physiological states of the fractions involved-for instance, to what extend the phases are related to the cell cycle or to cell communications. Cell fractions are assumed to be strongly related to the individual cell cycle in the works [4], [8], [9]. Our biological interpretations are closer to those in [13]; there an interesting structured threephase mathematical model stressing on the enzymatic competence of the cells is proposed and discussed.

\section{B. Relation to enzyme kinetics}

Once postulating that cell growth is tightly related to enzyme activity of the cells, then it is natural to make use of basic enzyme kinetics in the modelling of cell growth. From the perspective of cell growth dynamics bacteria can be viewed as complexes of enzymes processing nutrient substrates. Thus cells are similar to enzymes in that both process specific substrates. The only big difference between cells and enzyme complexes is the reproduction property that does not exist in the world of enzymes (viewed as chemical substances).

We recall some familiar knowledge from enzyme kinetics. The Michaelis-Menten differential equation describing the uptake of a substrate by a specific enzyme

$$
d s / d t=- \text { const } s /\left(K_{m}+s\right)
$$

will be briefly referred as the MM-model. The MMmodel is used to represent Monod specific growth rate function $\mu(s)=$ const $s /\left(K_{m}+s\right)$ which is an integral part of various classical cell growth models [11]. For the construction of our models we make use of the familiar reaction scheme between an enzyme $E$ with a single active site and a substrate $S$, forming an enzymesubstrate complex $C$, which then yields product $P$ :

$$
S+E \underset{k_{-1}}{\stackrel{k_{1}}{\rightleftarrows}} C \stackrel{k_{2}}{\longrightarrow} P+E
$$

We shall call this scheme Henri-Michaelis-Menten (HMM) reaction scheme in tribute to the prominent scholars Victor Henri, Leonor Michaelis and Maud Menten [5], [6], [10], cf. also [14],[16]. The HMMscheme leads to the familiar enzyme kinetics model involving four phase variables: the concentrations of the substrate $S$, the product $P$ and the concentrations of the two forms of the enzyme-free $E$ and bound $C$. Denoting the concentrations: $s=[S], \quad e=[E], \quad c=$ $[C], \quad p=[P]$, and applying the mass action law we obtain a system of four ODE's:

$d s / d t=-k_{1} e s+k_{-1} c, d e / d t=-k_{1} e s+\left(k_{-1}+k_{2}\right) c$, $d c / d t=k_{1} e s-\left(k_{-1}+k_{2}\right) c, d p / d t=k_{2} c$,

to be further called the HMM-model.

In contrast to the MM-model the HMM-model describes not only the dynamics of the concentration of the substrate $S$, but also the dynamics of the concentrations of the two fractions of the enzyme-free $E$ and bound $C$ plus the product $P$. Knowing that the solution of the MM-model is an approximation of the solution for the substrate in the HMM-model [12], it is natural to come to the idea to use the exact HMM-model-instead of the approximate MM-model-when constructing a cell growth model.

The idea of using the HMM-mechanism for modelling cell growth requires to introduce phase variables corresponding to certain fractions of the cell population which we relate to cell phases. Our modelling approach to bacterial growth has been explained in some detail 
in [1], where some initial variants of structured twophase models are proposed. Here we revisit some of these models by basing them more tightly on the HMM reaction scheme, resp. HMM-model.

Let us briefly sketch the idea of our modelling approach. Recall that in a familiar classical cell growth model the utilization of the substrate $s$ by the cell biomass $z$ is described by: $d s / d t=-\delta \mu z$, where $\mu$ is Monod specific growth rate function $\mu(s)=$ const $s /(K+s)$ [11]. If the cell biomass $z$ is nearly constant - as is usually the desired case under a continuous cultivation process - then this model coincides with the MM-model for the substrate uptake by corresponding enzymes. Several remarks can be made. First, the connection between the two models is obvious as substrate nutrients are utilized by the cell via corresponding enzyme complexes in the cell cytoplasm; this explains why classical Monod type models are so successful and popular. Second, recall that the MM-model is an approximation of the accurate HMM-model, hence classical models are likely to be approximations of certain more accurate models involving additional phase variable(s). Third, we know that the above mentioned approximation is a good one only if the ratio enzyme/substrate is small, which is rarely the case when realistic cell growth is considered [15]. Fourth, passing from the MM-model to the more general HMM-model we get free of rational functions in the right hand side of the model such as $s /(K+s)$ (and have only simple polynomial expressions), however, for the expense of introducing additional phase variable(s). Having in mind these remarks one can ask what would be the natural way to upgrade classical cell growth models in order to make them more adequate.

To answer this question we propose suitable reaction schemes for the cell growth dynamics based on the HMM-model. This paper focuses on cell growth models related to batch cultivation. Continuous cultivation models are proposed and studied in a forthcoming work.

\section{Model assumptions}

There may be various biological interpretations of the phases depending on the particular situations (bacterial species, nutrient substrate, environmental conditions etc). The following interpretations seem plausible in certain conditions.

We start with the following abstractions. Enzyme complexes are considered as the most important components of the cells (from the perspective of their production abilities). Apart of enzymes bacterial cells contain other substances such as specific proteins, nucleotides, water, minerals etc. For the purposes of this study we shall consider these substances as playing auxiliary role in the metabolic processes. We assume that the total biomass of the bacteria is proportional to the total mass of enzymes in the bacteria cells. Hence, in cell growth dynamics bacteria can be considered as organized complexes of enzymes that perform specific metabolic functions generalizing the functions of particular enzymes. As already mentioned the functions of specific enzymesubstrate pairs are modelled by enzyme kinetics systems of ODE's, e. g. the HMM-model. Considered as sets of enzymes, cells can also be modelled by the enzyme kinetics systems of ODE's.

The total microbial biomass increases for the expense of the utilized nutrient substances and the product substances produced by the bacteria. From an abstract perspective, proteins reproduce themselves-via the ribosome mechanisms. Part of the newly built products "come into life" as newborn cell components. For simplicity we may assume that one of the newly formed daughter cells coincides with the mother cell (which corresponds to reproduction by cellular budding).

Classical bacterial growth models typically make use of a single variable for the biomass concentration. The use of a single variable for the biomass does not permit to distinguish cells of different physiological states at a given moment $t$. In this work we distinguish between two main phases of bacterial cells:

- cells in lag phase denoted as $X$-cells;

- cells in log phase denoted as $Y$-cells.

Let us recall some characteristics of these two phases. Lag phase $X$-cells do not reproduce and their metabolic activity is limited. $X$-cells appear as dormant being stressed by environmental perturbations, nutrient limitation etc. The moment when $X$-cells start to be metabolically active by consuming nutrient substrates and forming products $P$, then we consider these cells as log phase $Y$-cells, that is $X$-cells transform into $Y$-cells.

$Y$-cells are metabolically active; they consume nutrient substrate $S$ from the environment to form product $P$. The latter can be viewed as the set of all biochemical substances (like proteins, nucleotides, etc) needed for the cell functioning, including reproduction. We assume that $Y$-cells are in reproduction state at the current moment. Reproduction can be viewed as part of the production process; since the newly formed substances $P$ are component part of the newborn cells we assume that this part of $P$ transforms into new cells. 
After reproduction (by binary fission) the newborn daughter cells are considered as Y-cells [7]. Thus newly built metabolic products become live $Y$-cells. The ultimate goal of the cell activities is cell reproduction. Log phase $Y$-cells are those that have reached this goal, in contrast to lag phase $X$-cells that are in an apparently dormant phase.

Both fractions of $X$ - and $Y$-cells are assumed to be homogeneously distributed (stirred) in the volume, so that we can work with their biomass concentrations (population densities) $x, y$, respectively. The same refers to the substances $S$ and $P$ which are also considered well stirred.

In agreement with the above assumptions we assign the following biological/biochemical meanings to the variables used in the proposed model:

$s=s(t)$ is the concentration of all nutrient substrates $S$ in the bioreactor (fermenter) at moment $t$ that are used for the bacterial growth;

$x=x(t)$ is the biomass (concentration or population) of $X$-cells being in lag phase at time $t$; these bacteria are not metabolically active. $X$-cells do not consume $S$ and do not produce $P$, neither they reproduce at time $t$. (Nevertheless, $x$ is not constant, due to transition of the phases.)

$y=y(t)$ is the biomass concentration of $Y$-cells in log phase at time $t ; Y$-cells utilize product $S$ and produce substances $P$. $Y$-cells are in the state of reproduction at time $t$. The substances metabolized by the $Y$-cells are part of the cell products denoted $P$.

$z=z(t)=x+y$ is the total biomass concentration that is the sum of the two fractions $x=x(t)$ and $y=$ $y(t)$.

$p=p(t)$ is the concentration of all product substances $P$ metabolized by the $Y$-cells including those excreted and those build up for growth and reproduction. Products $P$ are partly included in the newborn daughter cells.

The above model assumptions, abstractions and interpretations suggest direct relations to enzyme kinetics. Thus $X$-cells can be related to free enzymes, as they are not involved in production or reproduction at a given time moment $t ; Y$-cells can be related to bound enzymes, as their enzyme complexes are actively engaged with production and reproduction at time $t$.

Our biological assumptions are close to the ones discussed in [13], [4], [8], [9]. Our proposed models are also based on biochemical arguments related to enzyme kinetics. However, in contrast to the cited works, we make use of the Henri-Michaelis-Menten mechanism involving the concentrations of substrate, enzymes (free and bound) and product (and not just of the single Michaelis-Menten differential equation for the substrate).

\section{Reaction steps of the cell growth process}

In the course of model construction we formulate certain possible reaction steps of the cell growth process, e. g.:

- cell growth reduces or decreases owing to limitation of nutrient substrate; cells stop product formation and stop reproducing themselves;

- cell growth increases due to abundance and utilization of nutrient substrate;

- cell population rapidly increases due to reproduction (by binary fission);

- transition of substrate into product via cell metabolic enzymes;

- transition of metabolized product into living cells;

- excretion of waste products of cell metabolism;

- death and disintegration of living cells;

- cell growth inhibition due to excess of substrate.

Some of the above reaction steps are related to the transition of cells from one phase to another. Especially important are the transitions from lag to log phase and vice versa. The last two steps concern the stationary and death phases. These two phases are also significant, but in this work we try to ignore them for the sake of simplicity, mainly focusing on the reaction steps involving lag-log transitions.

\section{BATCH CULTIVATION: REACTION SCHEMES}

\section{A. The lag-log cell phase transition}

We shall borrow the basic lag-log cell phase transition reaction scheme from Henri-Michaelis-Menten enzyme kinetic [12], [14], identifying free enzymes with $X$ cells (bacteria in lag phase) and bound enzymes with $Y$-cells (log phase bacteria). Following the familiar reaction scheme for the substrate-enzyme kinetics when the enzymes possess a single active site we have:

$$
S+X \underset{k_{-1}}{\stackrel{k_{1}}{\rightleftarrows}} Y \stackrel{k_{2}}{\longrightarrow} P+X
$$

wherein $k_{1}, k_{-1}, k_{2}$ are rate constants.

Passing from a model describing a specific substrateenzyme reaction to a model describing a substrate-cell activity is a jump from molecular level to cell population level. A possible justification of such a jump is that: 
i) cells can be viewed as (organized) complexes of enzymes, ignoring thus other components of the cells; ii) nutrient substrates can be restricted to a few (sometimes a single) limiting substrate(s); iii) the cell enzymes process the nutrient substrate $S$ to produce new complex substances (like proteins, via the ribosome mechanism) partly included in product $P$ in the right hand-side of reaction scheme (RSP); iv) $X$-cells that become engaged in metabolic/reproductive processes transform (change their status)from lag into $\log$ phase $Y$-cells.

The product $P$ in the scheme (RSP) can be viewed as (part of) the highly organized substances needed to complete the enzymatic competence of the cell to be able to reproduce. We can think of the $Y$-cells as having reached the highest inner protein-nucleotide organization needed to reproduce. We shall thus assume that the $Y$ cells are those engaged in production and reproduction at the current moment $t$, in contrast to $X$-cells that do not (re)produce at moment $t$.

In the scheme (RSP) the $Y$-cells correspond to bound enzymes $C=E-S$ producing $P$. A formal analogy between the $S-E$ pairs and the $S-X$ pairs is that an $S-E$ pair is an enzyme in a special temporal state (bound enzyme, engaged in a product formation process) and similarly a $S-X$ pair is a cell in a temporal state (occupied in a (re)production process).

The (RSP) reaction scheme represents three reaction steps in the cell growth process, involving the two $X \rightleftarrows Y$ transitions plus the $S \longrightarrow P$ transformation. The scheme shows that $Y$-cells are entirely dependant on the available nutrient substrate $S$. Indeed, the presence of $S$ stimulates (initiates) the transition of lag phase $X$ cells into $\log$ phase $Y$-cells. On the other side, substrate limitation leads to a decrease (up to disappearance) of $Y$-cells, resp. of product formation (including reproduction). The reverse transition $X \longleftarrow Y$ can be interpreted as the case when $X$-cells absorb a certain amount of nutrient substrate but then do not process it (and excrete back the substrate). This reaction step seems to have minor impact on the total cell growth process.

Applying the mass action law to reaction scheme (RSP) leads to the following system of ordinary differential equations:

$$
\begin{aligned}
d s / d t & =-k_{1} x s+k_{-1} y \\
d x / d t & =-k_{1} x s+k_{-1} y+k_{2} y \\
d y / d t & =k_{1} x s-k_{-1} y-k_{2} y \\
d p / d t & =k_{2} y
\end{aligned}
$$

familiar from enzyme kinetic textbooks [12]. Appropriate initial conditions corresponding to a batch cultivation process are: $s(0)=s_{0}, x(0)=x_{0}, y(0)=y_{0}, p(0)=$ 0 .

The terms with $k_{-1}$ in the right hand-side of (1) can be suppressed as $k_{-1}$ is expected to be small, then we obtain a simpler model:

$$
\begin{aligned}
d s / d t & =-k_{1} x s \\
d x / d t & =-k_{1} x s+k_{2} y \\
d y / d t & =k_{1} x s-k_{2} y \\
d p / d t & =k_{2} y
\end{aligned}
$$

corresponding to the reaction scheme:

$$
S+X \stackrel{k_{1}}{\longrightarrow} Y \stackrel{k_{2}}{\longrightarrow} P+X,
$$

Systems (1), (2) modelize a batch mode bioreactor under the assumption that cells do not reproduce nor die. The basic models (1), (2) take into account substrate limitation in so far that only the log phase biomass declines due to substrate depletion; however the total biomass concentration $z=x+y=$ const remains constant in spite of substrate depletion. As in enzyme kinetics here we see again the conservation law for the substrate $d s / d t+d y / d t+d p / d t=0$ as well. A realistic cell growth model should include the above mentioned additional reaction steps, such as reproduction and mortality in order to encompass the complete cell growth process.

\section{B. Reproduction}

Reproduction can be viewed as the most important part of the total "production" process-during reproduction a mother cell produces two daughter cells, thus one more cell appears as result of this process.

Reproduction: a simple reaction scheme. $Y$-cells are engaged in formation (biosynthesis) of products $P$, which are important components of newborn cells. Thus we may view at reproduction as part of the production process. We assume that: a) cell growth is mainly due to reproduction-hence to $Y$-cells; b) $Y$-cells utilize product $P$ to reproduce; c) newborn cells are $Y$-cells, that is newborn cells do reproduce (by binary fission) [7]. Following these assumptions a simple reaction scheme for the growth-reproduction process is:

$$
P+Y \stackrel{k_{3}}{\longrightarrow} 2 Y \text {. }
$$

From a "mechanistic" point of view reaction scheme (RSG) can be interpreted as follows: the mother $Y$-cell transforms into one of the daughter cells, while the other 
daughter cell is built by means of product $P$ components. In other words, a certain part of product $P$ "comes into life" as a newborn cell. Putting reaction schemes (RSP) and (RSG) together:

$$
\begin{gathered}
S+X \underset{k_{-1}}{\stackrel{k_{1}}{\rightleftarrows}} Y \stackrel{k_{2}}{\longrightarrow} P+X, \\
P+Y \stackrel{k_{3}}{\longrightarrow} 2 Y,
\end{gathered}
$$

and applying the mass action law, we obtain the model:

$$
\begin{aligned}
d s / d t & =-k_{1} x s+k_{-1} y \\
d x / d t & =-k_{1} x s+k_{-1} y+k_{2} y \\
d y / d t & =k_{1} x s-k_{-1} y-k_{2} y+k_{3} p y \\
d p / d t & =k_{2} y-k_{3} p y
\end{aligned}
$$

with initial conditions (corresponding to a batch cultivation process): $s(0)=s_{0}, x(0)=x_{0}, y(0)=y_{0}, p(0)=$ 0 .

Respectively, assuming $k_{-1}=0$ we obtain the simpler model:

$$
\begin{aligned}
d s / d t & =-k_{1} x s \\
d x / d t & =-k_{1} x s+k_{2} y \\
d y / d t & =k_{1} x s-k_{2} y+k_{3} p y \\
d p / d t & =k_{2} y-k_{3} p y
\end{aligned}
$$

induced by the reaction scheme:

$$
\begin{gathered}
S+X \stackrel{k_{1}}{\longrightarrow} Y \stackrel{k_{2}}{\longrightarrow} P+X, \\
P+Y \stackrel{k_{3}}{\longrightarrow} 2 Y .
\end{gathered}
$$

(RSPs)-(RSG)

\section{Comparison to classical models}

We next compare model (3) with the classical model

$$
\begin{aligned}
& d s / d t=-\delta \mu(s) z \\
& d z / d t=\delta \mu(s) z \\
& \mu(s)=s /\left(K_{m}+s\right) .
\end{aligned}
$$

Proposition. Classical model (5) is a special case of model (3) under the assumption that the biomass $x$ and the product $p$ are (nearly) constant during the process.

Proof. We sum up the second and the third equations in (3) to obtain a relation for the total biomass $z=x+y$ :

$$
d z / d t=d x / d t+d y / d t=k_{3} p y .
$$

Integrating in the interval $[0, t]$ we have

$$
z=x+y=x_{0}+y_{0}+k_{3} I, \quad I(t)=\int_{0}^{t} p(\tau) y(\tau) d \tau .
$$

Let us assume that the biomass $x$ is at a steady state:

$$
d x / d t=-k_{1} x s+k_{-1} y+k_{2} y=0 .
$$

From (3) this implies $d s / d t=-k_{2} y$. Substituting $x$ by $z-y$ we have

$$
-k_{1} x s+k_{-1} y+k_{2} y=-k_{1}(z-y) s+k_{-1} y+k_{2} y=0,
$$

which gives

$$
y=s z /\left(K_{m}+s\right), \quad K_{m}=\left(k_{-1}+k_{2}\right) / k_{1} .
$$

Substituting the above expression for $y$ in $d s / d t=$ $-k_{2} y$ we have

$$
d s / d t=-k_{2} s z /\left(K_{m}+s\right),
$$

which gives the first equation of (5): $d s / d t=-\delta \mu(s) z$ with $\delta=k_{2}$.

To obtain the second equation of (5) we substitute $y=s z /\left(K_{m}+s\right)$ in the last equation of $(3): d p / d t=$ $k_{2} y-k_{3} p y$ to obtain (using $d z / d t=k_{3} p y$ ):

$$
d p / d t=k_{2} z s /\left(K_{m}+s\right)-d z / d t
$$

or

$$
d z / d t=k_{2} z s /\left(K_{m}+s\right)-d p / d t .
$$

Assuming a constant production of $p$, i. e. approximately $d p / d t=0$, we arrive at the classical model (5) for the substrate consumption and the biomass dynamics in a batch reactor.

Remark. Note that if the biomass is nearly constant, i. e. $z=z_{0}$, then the ODE for the consumption of $s$ in (5) turns into the Michaelis-Menten ODE of the substrate uptake in a chemostat: $d s / d t=-k_{2} z_{0} s /\left(K_{m}+s\right)$.

Proposition 1 shows that the basic Monod model (5) is an approximation of model (3) under the assumption of (nearly) constant biomass and product. In other words, model (3) generalizes model (5).

\section{Mortality}

To model the process of cell mortality, we first should decide which cell fractions are most susceptible of dying. One possibility is to introduce a separate cell fraction, as done in [13]. In this work we shall try to keep our model mathematically simple and decide against introducing a new cell fraction, resp. a new phase variable. From the two fractions $X$ and $Y$ we have to choose which one is more likely to die. In this work we accept that the $X$-cell fraction is the one that is more likely to be affected (but we leave this decision open for future examination). We thus accept here that only $X$-cells die and disintegrate. In addition, we assume that disintegrated cells transform partially to substrate $S$ and partially to product $P$ :

$$
X \stackrel{k_{s}}{\longrightarrow} S, \quad X \stackrel{k_{p}}{\longrightarrow} P .
$$

Putting all three reaction schemes (RSP), (RSG) and (RSD) together we have: 


$$
S+X \underset{k_{-1}}{\stackrel{k_{1}}{\rightleftarrows}} Y \stackrel{k_{2}}{\longrightarrow} P+X
$$

$P+Y \stackrel{k_{3}}{\longrightarrow} 2 Y, \quad X \stackrel{k_{s}}{\longrightarrow} S, \quad X \stackrel{k_{p}}{\longrightarrow} P$. wherein $k_{1}, k_{-1}, k_{2}, k_{3}$ and $k_{s}, k_{p}$ are rate constants.

Applying the mass action law the above global scheme leads to the dynamical model

$$
\begin{aligned}
d s / d t & =-k_{1} x s+k_{-1} y+k_{s} x \\
d x / d t & =-k_{1} x s+k_{-1} y+k_{2} y-\left(k_{s}+k_{p}\right) x \\
d y / d t & =k_{1} x s-k_{-1} y-k_{2} y+k_{3} p y \\
d p / d t & =k_{2} y-k_{3} p y+k_{p} x
\end{aligned}
$$

wherein the parameters $k_{1}, k_{-1}, k_{2}, k_{3}$ and $k_{s}, k_{p}$ are rate constants.

Biological meaning of the terms in the models. The reaction schemes make the biological interpretation of the terms in model (6) almost obvious. Here they are:

$k_{1} x s$ represents the consumption of $S$ by bacteria $X$ and the transition of bacteria $X$ into bacteria $Y$;

$k_{-1} y$ describes an amount of substrate concentration temporal;y stored by the cells but not further processed;

$k_{2} y$ describes the amount of product concentration formed by $Y$-cells and the transition of $Y$-cells into $X$ cells;

$k_{3} p y$ describes the increase of the cell population due to reproduction and the decrease of $P$ due to its transition to newly formed $Y$-cells (reaction scheme (RSG));

$k_{s} x$ describes the decay of bacteria $X$ and the disintegrated part of the dead cells transforming into substrate $S$-reaction scheme (RSD);

$k_{p} x$ describes the decay of bacteria $X$ and the disintegrated part of the dead cells transforming into product $P$-reaction scheme (RSD).

The parameters have the meaning of specific rate constants as follows: $k_{1}$ is the substrate utilization rate, $k_{-1}$ is the substrate non-utilization rate, $k_{2}$ is the production rate, $k_{3}$ is the reproduction rate, $k_{s}$ and $k_{p}$ are death rates.

Whenever appropriate we may also use specific reaction steps describing waste product excretion, e. $\mathrm{g}$.

$$
P+Y \stackrel{\delta}{\longrightarrow} Q
$$

implying corresponding terms $\delta p y$ in the dynamical system.

We finish this section with the following hypothesis:

Hypothesis. Cell growth models for batch cultivation can be based on reaction schemes, involving reaction steps such as (RSG), (RSP), (RSD), (RSW).

\section{COMPUTER EXPERIMENTS}

We present the results of two computer experiments based on the above reaction schemes. For the computer experiments real experimental data for the biomass and product concentrations have been used. The graphics of the solutions demonstrate a good fit to the experimental data. In particular the lag phase data for the biomass are fitted better than when classical models such as (5) are used.

Computer experiment 1. The model is:

$$
\begin{aligned}
d s / d t & =-k_{1} x s+k_{-1} y \\
d x / d t & =-k_{1} x s+k_{-1} y+k_{2} y \\
d y / d t & =k_{1} x s-k_{-1} y-k_{2} y+\beta p y \\
d p / d t & =k_{2} y-\gamma p y
\end{aligned}
$$

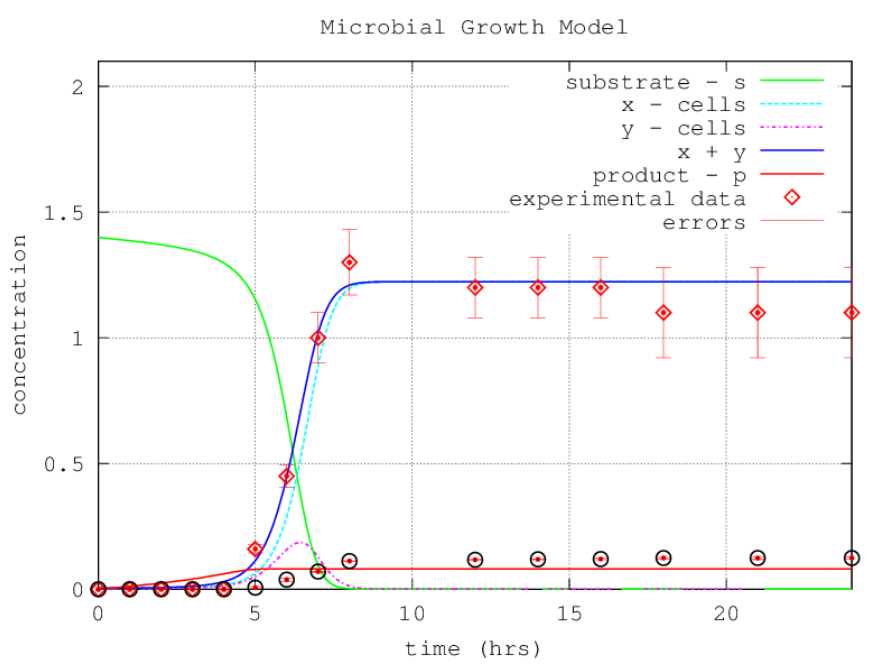

Fig. 1. Numerical solution of cell growth model (7) using Matlab

The values of the parameters used in model (7) are as follows: $k_{1}=3.48691, k_{-1}=0, k_{2}=7, \beta=$ 41.5095, $\gamma=86.4424 ; s_{0}=1.4, x_{0}=0.00450748$. The graphs of the solutions are represented in Fig. 1.

Model (7) is close to model (6) but is without mortality terms and there we have $\beta \neq \gamma$. To explain why in this experiment $\beta<\gamma$ we need to introduce a waste product excretion step, as done in the next experiment.

Computer experiment 2. The model is as follows:

$$
\begin{aligned}
& d s / d t=-k_{1} x s+k_{-1} y \\
& d x / d t=-k_{1} x s+k_{-1} y+k_{2} y-k_{d} x \\
& d y / d t=k_{1} x s-k_{-1} y-k_{2} y+(\alpha-\delta) p y \\
& d p / d t=k_{2} y-(\alpha+\delta) p y+k_{d} x
\end{aligned}
$$


The reaction scheme leading to model (8) involves a decay step (RSD) and a waste product excretion step (RSW) as follows:

$$
\begin{gathered}
S+X \underset{k_{-1}}{\stackrel{k_{1}}{\longrightarrow}} Y \stackrel{k_{2}}{\longrightarrow} P+X, \\
P+Y \stackrel{\alpha}{\longrightarrow} 2 Y, \\
P+Y \stackrel{\delta}{\longrightarrow} Q, \\
X \stackrel{k_{d}}{\longrightarrow} P,
\end{gathered}
$$

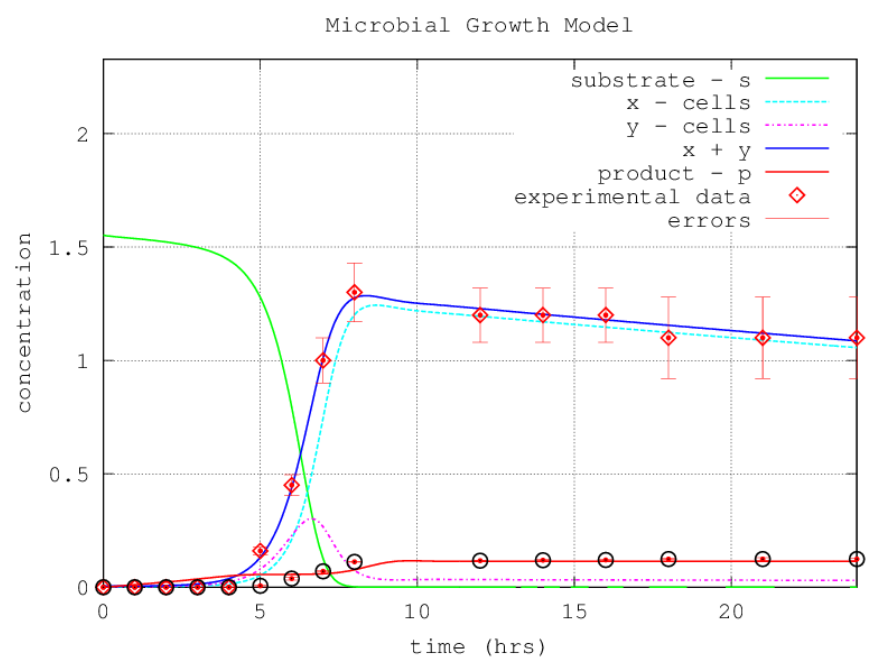

Fig. 2. Numerical solution of cell growth model (8) using Matlab

wherein $Q$ represents waste product. The values of the parameters used in model (8) are as follows: $k_{1}=$ $4.18191, k_{-1}=0, k_{2}=4.50413, \alpha=60.6611, \delta=$ $21.3493, k_{d}=0.136806 ; s_{0}=1.55196, x_{0}=$ 0.00490581 . The graphs of the solutions fitting real experimental data are visualized in Fig. 2. A very good fit can be observed.

\section{CONCLUSION}

In this work we formulate certain reaction steps in the cell growth process and try to base our models entirely on proposed reaction schemes. Our reaction schemes for the phase transition of the cells make use of appropriate reaction schemes from enzyme kinetics. Elements of the Michaelis-Menten enzyme kinetics can be observed in classical cell growth models using the Monod specific growth rate function $\mu$. In this paper instead of the (approximate) Michaelis-Menten enzyme kinetics for the substrate uptake we use the (exact) Henri-MichaelisMenten enzyme kinetics (HMM-reaction mechanism).

It has been noted in the literature that classical Monod type models often fail to describe adequately bio-reactors under perturbed conditions. It has been also recognized that Monod models lack (do not fit well) the lag phase of the cells. Our numerical experiments make us believe that structured models provide more flexibility and can be better fitted to real data. The use of reaction schemes makes the construction of particular cell growth models simple and instructive; it also contributes to understanding the underlying biological mechanism. Our hypothesis is that cell growth models can be entirely based on reaction schemes.

\section{ACKNOWLEDGEMENTS}

The author is indebted to Prof. V. Beschkov from the Institute of Chemical Engineering, BAS, and Prof. M. Kamburova from the Institute of Microbiology, BAS, for providing real experimental data on microbial growth and for useful discussions on the behavior of the microorganisms and the proposed reaction schemes.

\section{REFERENCES}

[1] Alt, R., S. Markov, Theoretical and computational studies of some bioreactor models, Computers and Mathematics with Applications 64 (2012), 350-360. http://dx.doi.org/10.1016/j.camwa.2012.02.046

[2] Droop, M., Vitamin B12 and marine ecology. IV. The kinetics of uptake, growth and inhibition in Monochrysis Lutheri. Journal of Marine Biology 48, 689-733.

[3] Gerber, M., R. Span, An Analysis of Available Mathematical Models for Anaerobic Digestion of Organic Substances for Production of Biogas, proc. IGRC, Paris 2008.

[4] Gouzé, J.-L., V. Lemesle, Two simple growth models in the chemostat. ARIMA 9, 145-155 (2008).

[5] Henri V., Recherches sur la loi de laction de la sucrase. $C$. $R$. Hebd. Acad. Sci., 133, 891-899 (1901).

[6] Henri V., Ueber das Gesetz der Wirkung des Invertins. Z. Phys. Chem., 39 (1901), 194-216.

[7] Jocelyn E. Krebs, Elliott S. Goldstein, Stephen T. Kilpatrick, Lewin's GENES XI, Jones \& Bartlett Publ., 2014.

[8] Lemesle, V., J.-L. Gouzé, A simple unforced oscillatory growth model in the chemostat. Bulletin of Mathematical Biology 70, 344-357 (2008). http://dx.doi.org/10.1007/s11538-007-9254-5

[9] Lemesle, V., J-L. Gouzé, A biochemically based structured model for phytoplankton growth in the chemostat. Ecological Complexity 2, 21-33 (2005). http://dx.doi.org/10.1016/j.ecocom.2004.08.001

[10] Michaelis, L., M. L. Menten. Die Kinetik der Invertinwirkung. Biochem. Z. 49, (1913), 333-369.

[11] Monod, J., The growth of bacterial cultures, Annual Reviews of Microbiology 3, 371-394 (1949). http://dx.doi.org/10.1146/annurev.mi.03.100149.002103

[12] Murray J. D., Mathematical Biology: I. An Introduction, Third Edition, Springer, 2002. 


\section{S Markov, Cell Growth Models Using Reaction Schemes: Batch Cultivation}

[13] Sissons, C. J., M. Cross, S. Robertson, A new approach to the mathematical modelling of biodegradation processes, Appl. Math. Modelling 10 (1986), 33-40. http://dx.doi.org/10.1016/0307-904X(86)90006-5

[14] Schnell, S., Chappell, M. J., Evans, N. D., M. R. Roussel, The mechanism distinguishability problem in biochemical kinetics: The single-enzyme single-substrate reaction as a case study. C. R. Biologies 329, 51-61 (2006). http://dx.doi.org/10.1016/j.crvi.2005.09.005
[15] Schnell, S., P. K. Maini, Enzyme kinetics at high enzyme concentration, Bulletin of Mathematical Biology 62, 483-499 (2000). http://dx.doi.org/10.1006/bulm.1999.0163

[16] Schnell, S., P. K. Maini, A century of enzyme kinetics: Reliability of the $K_{M}$ and $v_{\max }$ estimates, Comments on Theoretical Biology 8, 169-187 (2003).

DOI: $10.1080 / 08948550390206768$ 